\title{
TAXAS DE CRESCIMENTO DE MUDAS DE CATINGUEIRA SUBMETIDAS A DIFERENTES SUBSTRATOS E SOMBREAMENTOS ${ }^{1}$
}

Bárbara França Dantas², Armando Pereira Lopes², Fabrício Francisco Santos da Silva ${ }^{3}$, Aldenir Alves Lúcio $^{2}$, Patrício Ferreira Batista ${ }^{4}$, Mayara Milena Menezes da Luz Pires ${ }^{5}$ e Carlos Alberto Aragão ${ }^{6}$

\begin{abstract}
RESUMO - A catingueira (Caesalpinia pyramidalis Tul.) é uma espécie nativa da caatinga dotada de grande resistência à seca e de grande potencial econômico. No entanto, não existem informações sobre o desenvolvimento dessa espécie em diferentes substrates e luminosidade, fatores ambientais que mais influenciam a produção de seedlings florestais. Dessa forma, este trabalho teve por objetivo avaliar o efeito de diferentes níveis de shading e tipos de substrates nas taxas de crescimento de mudas de catingueira. O experimento foi conduzido na Universidade do Estado da Bahia e na Embrapa Semiárido, Petrolina, PE. As sementes foram semeadas em recipientes preenchidos com diferentes substratos: solo, areia, solo+areia, solo+areia+esterco caprino e substrato comercial, sendo os recipientes mantidos em quatro diferentes sombreamentos, a céu aberto (sem sombreamento) e em telados tipo sombrite de 30\%, 50\% e 75\% de sombreamento. Aos 30, 60 e 100 dias após a estabilização da emergência das plântulas, as mudas foram avaliadas quanto à taxa de crescimento absoluto, taxa de crescimento relativo, taxa assimilatória líquida, razão de área foliar,área foliar específica, peso específico da folha e razão de peso foliar. Não houve interação significativa entre os dois fatores estudados em nenhuma das taxas de crescimento calculadas. Nas condições deste trabalho, foi possível concluir que as taxas de crescimento das mudas de catingueira, em geral, não foram influenciadas pela luminosidade às quais elas foram submetidas e que solo coletado em região de caatinga, combinado ou não, com areia e esterco, possibilitou maior crescimento das mudas.
\end{abstract}

Palavras-chave: Caesalpinia pyramidalis, luminosidade e caatinga.

\section{GROWTH RATES OF CATINGUEIRA SEEDLINGS SUBMITTED TO DIFFERENT SUBSTRATES AND SHADING}

\begin{abstract}
Caesalpinia pyramidalis Tul. is a native species from the caatinga biome, with a great resistance to drought and great economic potential. However, there is no information regarding the development of this species under different substrates and light intensities, the major environmental factors that influence forest seedling growth. The objective of this work was to evaluate the effect of different shadings and substrates on the growth rates of $\boldsymbol{C}$.pyramidalis seedlings. The experiment was carried out at the Universidade do Estado da Bahia and Embrapa Semi-Árido. The seeds were sowed in recipients filled with different substrates: soil, sand, soil+ sand, soil+ sand+ goat manure and commercial substrate. The recipients were maintained under different shadings: sunlight (no shading) and at 30\%, 50\% and 75\% shadings. 30,60 and 100 days after the emergence stabilization, the seedlings were evaluated as to the absolute growth rate, relative growth rate, net assimilation rate, leaf area ratio, specific leaf area, specific leaf weight and leaf weight ratio. There was
\end{abstract}

\footnotetext{
${ }^{1}$ Recebido em 18.06.2007 e aceito para publicação em 24.04.2009.

${ }^{2}$ Centro de Pesquisa Agropecuária do TrópicoSemi-ÁridodaEmpresa Brasileirade PesquisaAgropecuária. E-mail: <barbara@cpatsa.embrapa.br> . ${ }^{3}$ Programa de Pós-Graduação em Agronomia da Universidade Federal da Paraíba (UFPB). E-mail: <fabriciofrancisco2005@ig.com.br>.

${ }^{4}$ Programa de Pós-Graduação em Fitotecnia da Universidade Federal do Semi-Arido (UFERSA).

${ }^{5}$ Graduação em Engenharia Agronômica pela Universidade do Estado da Bahia (UNEB). E-mail: <milamlp@ gmail.com>.

${ }^{6}$ Departamento de Tecnologia e Ciências Sociais daUNEB. E-mail: <carlosaragao@ hotmail.com>.
} 
no interaction between the two studied factors for any growth rate. Under the conditions of this work, it is possible to conclude that, in general, the growth rates of C.pyramidalis seedlings were not influenced by shading and that the soil collected in the caatinga region, associated or not, to sand and manure, allowed a higher growth.

Keywords: Caesalpinia pyramidalis, luminosity and caatinga.

\section{INTRODUÇÃO}

O Brasil possui 385 milhões de hectares de florestas nativas (IPEF, 2005), tendo a região de Caatinga cerca de 800 mil km², o que corresponde a $11 \%$ do território nacional e $70 \%$ do território nordestino, abrangendo os Estados do Ceará, Rio Grande do Norte, Paraíba, Pernambuco, Sergipe, Alagoas, Bahia, sul e leste do Piauí e norte de Minas Gerais (LIMA, 1996). A caatinga tem fisionomia de deserto, com índices pluviométricos muito baixos, em torno de 500 a $700 \mathrm{~mm}$ anuais. A temperatura situa-se entre 24 e $26^{\circ} \mathrm{C}$ e varia pouco durante o ano. Além dessas condições climáticas rigorosas, a região das caatingas está submetida a ventos fortes e secos, que contribuem para a aridez da paisagem nos meses de seca (SAMPAIO e RODAL, 2000).

Entre as espécies nativas da caatinga, a catingueiraverdadeira (Caesalpinia pyramidalis Tul.) apresenta grande potencial econômico devido à sua rusticidade e ao seu aproveitamento madeireiro, ao potencial para reflorestamento, uso medicinal e, principalmente, à sua propriedade extrativa (OLIVEIRA, 1976).

As folhas de $C$. pyramidalis Tul. são empregadas para controle de febre, doenças estomacais e como diurético (BAHIA, 1979). Suas folhas, flores e casca são, também, usadas no tratamento de infecções catarrais e em diarréias e disenterias (NISHIZAWA et al., 1995). Hardesty et al. (1988) também conferiram a espécie propriedades antidiarréicas e potencial forrageiro, sendo uma das plantas sertanejas cujas gemas brotam nas primeiras manifestações de umidade. Dessa forma, no início do período das chuvas o gado procura as folhas jovens extremamente palatáveis, sendo, entre outras, preferidas por bovinos, caprinos e ovinos (HARDESTY et al., 1988). Após o início das chuvas, em alguns dias a folhagem dessa espécie libera um cheiro pungente, sendo desprezada pelo rebanho. As folhas, então, secam e caem no início da estação seca, tornando-se uma forragem nutritiva nesse período (PFEISTER e MALECHEK, 1986). Essa característica garante a disponibilidade de forragem de catingueira durante o período seco (HARDESTY et al., 1988). Segundo Nishizawa et al. (1995), essa espécie produz madeira para lenha, carvão e estacas, com densidade de 0,85 g.m ${ }^{-3}$ (ZAKIA et al., 1990)

Os fatores ambientais que mais influenciam a produção de mudas florestais são o substrato e a luminosidade. Substrato é o meio em que as raízes se desenvolvem, formando um suporte estrutural, fornecendo água, oxigênio e nutrientes para que a parte aérea das mudas se desenvolva (OLIVEIRA et al., 2005). Inúmeros substratos em sua constituição original ou combinada são usados atualmente para propagação de espécies florestais. Na escolha de um substrato, deve-se observar, principalmente, suas características físicas e químicas, a espécie a ser plantada, além dos aspectos econômicos como baixo custo e grande disponibilidade (FONSECA, 2001). De acordo com Oliveira et al. (2005), o substrato deve apresentar propriedades físicas e químicas adequadas para o desenvolvimento das plantas, sendo as físicas determinantes, por serem de difícil correção. O substrato deve ser leve para facilitar o manuseio e o transporte, apresentar boa porosidade, drenagem e capacidade de retenção de água, ser suficientemente consistente para fixar as plantas, isento de patógenos de solo, não conter sementes ou propágulos de plantas daninhas, não conter componentes de fácil decomposição, possuir composição uniforme para facilitar o manejo das plantas e apresentar um custo compatível com a atividade.

Negreiros et al. (2004) salientaram a conveniência da associação de materiais orgânicos, especialmente em mistura com o solo, para melhorar a textura do substrato e, dessa maneira, propiciar boas condições físicas e fornecer os nutrientes necessários ao desenvolvimento das raízes e da muda.

A luminosidade por sua vez controla os processos responsáveis pelo acúmulo de matéria seca, contribuindo para o crescimento das mudas. O estudo da luminosidade 
é fundamental para a avaliação do potencial dessas espécies em programas de revegetação, pois a disponibilidade de luz constitui um dos fatores críticos para o seu desenvolvimento (GAJEGO et al., 2001). A importância deste fator tem levado diversos autores a classificar as espécies florestais em grupos ecológicos distintos de acordo com a sua capacidade de adaptação às condições de luminosidade ambiental e cujo conhecimento é chave importante para a compreensão da dinâmica das florestas e seu manejo (AMO, 1985).

A análise de crescimento descreve as condições morfofisiológicas da planta em diferentes intervalos de tempo, permitindo acompanhar a dinâmica da produtividade. É um método a ser utilizado na investigação do efeito dos fenômenos ecológicos sobre o crescimento, como a adaptabilidade das espécies em ecossistemas diversos, efeitos de competição, diferenças genotípicas e efeito de práticas agronômicas sobre o crescimento (MAGALHÃES, 1986).

Em virtude da carência de conhecimentos, os estudos básicos para produção de mudas são de extrema importância para o desenvolvimento da atividade florestal e para programas de conservação. Assim, este trabalho teve por objetivo avaliar o efeito de diferentes níveis de sombreamento e de diferentes substratos nas taxas de crescimento de mudas de catingueira.

\section{MATERIAL E MÉTODOS}

O experimento foi conduzido no período de março a julho de 2006 em casas de vegetação, teladas com diferentes níveis de sombreamento, da Universidade do Estado da Bahia (UNEB), Juazeiro, BA, e no Laboratório de Sementes/Fisiologia Vegetal da Embrapa
Semiárido, Petrolina, PE.

As sementes de catingueira (Caesalpinea pyramidalis Tul.) foram coletadas de árvores em uma área de caatinga nos distritos de Juremal e Massaroca pertencentes ao Município de Juazeiro, BA, caracterizados por um clima semiárido, com temperaturas máxima, média e mínima anuais de $29,6{ }^{\circ} \mathrm{C}, 24,2{ }^{\circ} \mathrm{C}$ e $20,3{ }^{\circ} \mathrm{C}$, respectivamente. A precipitação média anual da região é de $399 \mathrm{~mm}$, com período chuvoso de novembro a março.

As sementes foram beneficiadas, com secamento das vagens em ambiente protegido e separação manual de sementes e material inerte, sendo determinado em seguida o teor de água das sementes. As sementes, cujo teor de água era de $9,46 \%$, foram semeadas, sem nenhum pré-tratamento, em sacos de polietileno de volume de 0,572 L, preenchidos com cinco diferentes substratos, sendo eles material de solo (S), areia (A), mistura de material de solo+areia $(1: 1, \mathrm{SA})$, mistura de material de solo+areia+esterco caprino (1:1:1, SAE) e substrato comercial, Plantimax ${ }^{\circledR}$ (SC). O material de solo foi coletado de área de caatinga no horizonte superficial de um Latossolo Vermelho-Amarelo. Os substratos não foram corrigidos quanto ao $\mathrm{pH}$, nem foi adicionado adubo químico a eles, sendo submetidos à análise química (Tabela 1).

Os recipientes foram mantidos em quatro diferentes sombreamentos, em céu aberto (sem sombreamento) e em telados tipo sombrite com $30 \%, 50 \%$ e $75 \%$ de sombreamento. Foram utilizadas cinco mudas (repetições) por tratamento, em um esquema fatorial $4 \times 5$ (sombreamentos e substratos), totalizando 100 parcelas para cada avaliação.

Tabela 1 - Análise química dos substratos avaliados Table1 - Chemical analysis of the substrates evaluated

\begin{tabular}{|c|c|c|c|c|c|c|c|c|c|c|c|c|c|}
\hline \multirow[t]{2}{*}{ Substrato } & \multicolumn{13}{|c|}{ Determinações } \\
\hline & $\begin{array}{l}\mathrm{MO} \\
\mathrm{g} / \mathrm{kg}\end{array}$ & $\mathrm{pH}$ & $\begin{array}{l}\text { C.E. } \\
\text { dS/m }\end{array}$ & $\begin{array}{c}\mathrm{P} \\
\mathrm{mg} / \mathrm{dm}^{3}\end{array}$ & $\mathrm{~K}$ & $\mathrm{Ca}$ & $\mathrm{Mg}$ & $\begin{array}{c}\mathrm{Na} \\
\mathrm{cmol}_{\mathrm{c}} / \mathrm{dm}^{3}\end{array}$ & Al & $\mathrm{H}+\mathrm{Al}$ & $\mathrm{S}_{\text {(bases) }}$ & CTC & $\begin{array}{l}\mathrm{V} \\
\%\end{array}$ \\
\hline$\overline{\mathrm{S}^{\mathrm{a}}}$ & 6,21 & 6,9 & 0,57 & 5 & 0,31 & 2,5 & 0,6 & 0,02 & 0,05 & 0,33 & 3,43 & 3,76 & 91 \\
\hline A & 0,83 & 8,3 & 0,29 & 17 & 0,09 & 1,5 & 0,3 & 0,02 & 0,00 & 0,00 & 1,91 & 1,91 & 100 \\
\hline AS & 5,79 & 7,6 & 0,55 & 49 & 0,23 & 2,5 & 0,6 & 0,01 & 0,00 & 0,00 & 3,34 & 3,34 & 100 \\
\hline SAE & 28,96 & 8,6 & 1,10 & 71 & 0,43 & 4,2 & 0,9 & 0,04 & 0,00 & 0,00 & 5,57 & 5,57 & 100 \\
\hline $\mathrm{SC}$ & 33,10 & 5,9 & 4,62 & 421 & 1,80 & 7,8 & 6,8 & 0,18 & 0,05 & 6,43 & 16,58 & 23,01 & 72 \\
\hline
\end{tabular}

${ }^{a} \mathrm{~S}=$ solo; $\mathrm{A}=$ areia; $\mathrm{AS}=$ solo+areia; $\mathrm{SAE}=$ solo+areia+esterco; e $\mathrm{SC}=$ substrato comercial.

${ }^{a} \mathrm{~S}=$ soil; $\mathrm{A}=$ sand $; \mathrm{AS}=$ soil+sand $\mathrm{SAE}=$ soil+sand+manure; and $\mathrm{SC}=$ commercial substrate. 
Aos 30,60 e 100 dias após a estabilização da emergência de plântulas (DEE), que ocorreu aos oito dias após a semeadura, as mudas foram avaliadas quanto à massa de matéria seca total e da folha (MMST e MMSF, respectivamente), área foliar (AF). A partir desses dados foram obtidos: taxa de crescimento absoluto (TCA), taxa de crescimento relativo (TCR), taxa assimilatória líquida (TAL), razão de área foliar (RAF), área foliar específica (AFE), peso específico da folha (PEF) e razão de peso foliar (RPF), de acordo com Benincasa (1988), calculados pelas seguintes expressões:

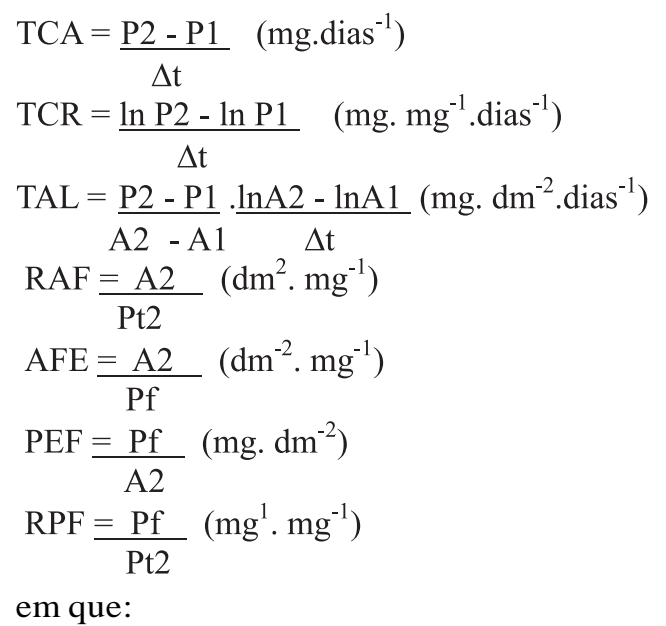

Pt1 = MMST da coleta anterior

$\mathrm{Pt} 2=$ MMST da coleta atual

$\mathrm{Pf}=\mathrm{MMSF}$ da coleta atual

$\mathrm{A} 1=\mathrm{AF}$ da coleta anterior

$\mathrm{A} 2=\mathrm{AF}$ da coleta atual

$\ln =\log$ aritmo neperiano

$\Delta \mathrm{t}=$ intervalo de tempo entre as coletas

O experimento foi disposto em delineamento inteiramente casualizado, em um esquema fatorial $4 \times 5$, sendo combinados tratamentos referentes a quatro sombreamentos e cinco substratos com quatro repetições. Os resultados foram submetidos à análise de variância e as médias, comparadas pelo teste de Tukey a $10 \%$ de probabilidade.

\section{RESULTADOS E DISCUSSÃO}

Na Tabela 1 são exibidos os resultados da análise química dos substratos utilizados, em que se verificou que os substratos com maiores teores de matéria orgânica, SAE e SC, apresentam os maiores valores de todas as determinações analisadas, exceto para o $\mathrm{pH}$, que foi o mais alto $(8,6)$ e o mais baixo $(5,9)$, respectivamente, em relação aos demais. Conforme Kämpf (2000), em substratos onde predomina a matéria orgânica; faixa ideal de $\mathrm{pH}$ recomendada é de 5,0 a 5,8 e, quando for à base de solo mineral, entre 6,0 e 6,5 .

As características químicas mais importantes nos substratos são: $\mathrm{pH}$ e a condutividade elétrica (SILVEIRA et al., 2002). Com relação ao pH, os substratos devem apresentar valores dentro de uma faixa considerada adequada para o cultivo de plantas, pois valores inadequados, além de influenciarem a disponibilidade de nutrientes (CARNEIRO, 1995), estão relacionados a desequilíbrios fisiológicos (WILSON, 1983).

De acordo com Oliveira (2005), a catingueira desenvolve-se melhor na presença de $\mathrm{Ca}, \mathrm{Mg}$ e $\mathrm{P}$, cujos maiores teores são verificados no $\mathrm{SC}$ e $\mathrm{pH}$ próximo à neutralidade, verificados nos substratos $\mathrm{S}$ e SA.

Observa-se na Tabela 2 que, entre os parâmetros avaliados, apenas o MMST1 obtido 30 DAEE apresentou interação entre os substratos e sombreamentos impostos. Não houve interação significativa entre os dois fatores estudados para nenhuma das taxas de crescimento calculadas. Apenas para MMST, MMSF, AF, TCA, TCR e RAF as mudas responderam, significativamente, ao sombreamento e para PEF não houve resposta significativa para nenhum dos tratamentos.

De acordo com Garcia (1989), para espécies florestais melhoradas como Pinus spp. e Eucaliptus spp. existe variação acentuada entre os valores médios de coeficiente de variação (CV) entre os diferentes parâmetros, determinando, assim, a necessidade de se considerar, na avaliação do coeficiente de variação, não só a variável em estudo, mas também a espécie e o tipo de experimentação instalado, além da idade de avaliação e número de repetições utilizados na experimentação. Além disso, esse autor afirmou que o coeficiente de variação $(\mathrm{CV})$, dependendo da variável em estudo, pode ser considerado muito alto quando apresenta valores acima de $90 \%$. Devido à grande variabilidade da espécie estudada algumas análises apresentaram $\mathrm{CV}$ alto e muito alto $(\mathrm{CV}>50 \%)$. Provavelmente devido a esse resultado, algumas das análises não apresentaram resultados significativos. 
Tabela 2 - Valores de F da análise de variância das taxas de crescimento de mudas de catingueira submetidas a diferentes sombreamentos e substratos

Table 2 - F Values of the variance analyses of the growth rates of the shootings of the "catingueira" submitted to different shadings and substrates

\begin{tabular}{|c|c|c|c|c|c|c|c|c|c|}
\hline Fonte de variação & $\mathrm{MMST}^{\mathrm{a}}$ & MMST2 & MMST3 & MMSF1 & MMSF2 & MMSF3 & AF1 & AF2 & AF3 \\
\hline Sombreamento & $3,468 * * b$ & $0,429 \mathrm{~ns}$ & $3,472 * *$ & $2,876 * *$ & $0,638 \mathrm{~ns}$ & $1,572 \mathrm{~ns}$ & $6,553 * * *$ & $2,647 *$ & $0.574 \mathrm{~ns}$ \\
\hline Substrato & $6,931 * * *$ & $9,671 * * *$ & $4,869 * * *$ & $8,798 * * *$ & $7,081 * * *$ & $4,960 * * *$ & $12,204 * * *$ & $5,615 * * *$ & $5.369 * * *$ \\
\hline Sombreamento*substrato & $2,232 * *$ & $0,803 \mathrm{~ns}$ & $1,246 \mathrm{~ns}$ & $0,846 \mathrm{~ns}$ & $1,194 \mathrm{~ns}$ & $1,184 \mathrm{~ns}$ & $1,249 \mathrm{~ns}$ & $1,081 \mathrm{~ns}$ & $1.291 \mathrm{~ns}$ \\
\hline CV\% & 48,69 & 30,48 & 42,89 & 64,60 & 42,01 & 59,10 & 33,76 & 41,35 & 57.44 \\
\hline Fonte de variação & TCA1 & TCA2 & TCR1 & TCR2 & TAL1 & TAL2 & RAF1 & RAF2 & RAF3 \\
\hline Sombreamento & $0,785 \mathrm{~ns}^{\mathrm{b}}$ & $2,980 * *$ & $2,272 *$ & $1,498 \mathrm{~ns}$ & $0,613 \mathrm{~ns}$ & $1,093 \mathrm{~ns}$ & $1,218 \mathrm{~ns}$ & $3,447 * *$ & $\overline{1,794 \mathrm{~ns}}$ \\
\hline Substrato & $3,161 * *$ & $2,325 *$ & $2,362 *$ & $1,693 \mathrm{~ns}$ & $1,607 \mathrm{~ns}$ & $3,077 * *$ & $6,273 * * *$ & $2,557 * *$ & $1,429 \mathrm{~ns}$ \\
\hline Sombreamento*substrato & $1,242 \mathrm{~ns}$ & $0,863 \mathrm{~ns}$ & $1,200 \mathrm{~ns}$ & $0,443 \mathrm{~ns}$ & $0,428 \mathrm{~ns}$ & $0,866 \mathrm{~ns}$ & $2,029 \mathrm{~ns}$ & $1,099 \mathrm{~ns}$ & $0,893 \mathrm{~ns}$ \\
\hline $\mathrm{CV} \%$ & 96,48 & 84,27 & 78,44 & 76,26 & 131,30 & 204,84 & 30,81 & 26,31 & 73,78 \\
\hline Fonte de variação & AFE1 & AFE2 & AFE3 & PEF1 & PEF2 & PEF3 & RPF1 & RPF2 & RPF3 \\
\hline Sombreamento & $0,890 \mathrm{~ns}$ & $0,456 \mathrm{~ns}$ & $1,196 \mathrm{~ns}$ & $0,540 \mathrm{~ns}$ & $0,790 \mathrm{~ns}$ & $1,956 \mathrm{~ns}$ & $1,616 \mathrm{~ns}$ & $0,881 \mathrm{~ns}$ & $1,712 \mathrm{~ns}$ \\
\hline Substrato & $1,706 \mathrm{~ns}$ & $2,244 *$ & $0,657 \mathrm{~ns}$ & $0,992 \mathrm{~ns}$ & $1,430 \mathrm{~ns}$ & $0,380 \mathrm{~ns}$ & $0,761 \mathrm{~ns}$ & $2,096 *$ & $4,036 * * *$ \\
\hline Sombreamento*substrato & $0,644 \mathrm{~ns}$ & $0,869 \mathrm{~ns}$ & $0,160 \mathrm{~ns}$ & $0,995 \mathrm{~ns}$ & $1,111 \mathrm{~ns}$ & $0,396 \mathrm{~ns}$ & $0,896 \mathrm{~ns}$ & $1,172 \mathrm{~ns}$ & $0,712 \mathrm{~ns}$ \\
\hline $\mathrm{CV} \%$ & 320,66 & 36,98 & 91,34 & 77,09 & 69,68 & 88,88 & 39,15 & 18,16 & 23,01 \\
\hline
\end{tabular}

a $\mathrm{TCA}=$ taxa de crescimento absoluto; $\mathrm{TCR}=$ taxa de crescimento relativo; $\mathrm{TAL}=$ taxa assimilatória líquida; $\mathrm{RAF}=$ razão de área foliar; $\mathrm{AFE}=$ área foliar específica; $\mathrm{PEF}=$ peso específico da folha; $\mathrm{RPF}=$ razão de peso foliar. ${ }^{\mathrm{b}} \mathrm{ns} ; \mathrm{e}^{*}, * * \mathrm{e} * * *=$ não significativo e significativo a $10 \%, 5 \%$, e $1 \%$, respectivamente.

a $\mathrm{TCA}=$ absolute growth rate; TCR = relative growth rate; TAL= liquid assimilatory rate; $\mathrm{RAF}=1 \mathrm{eaf}$ area ratio; $\mathrm{AFE}=\mathrm{specific}$ leaf area; $\mathrm{PEF}=$ leaf specific weight; $\mathrm{RPF}=$ leaf weight ratio. ${ }^{\mathrm{b}} \mathrm{ns} ;$ and $* * *$ and $* * *=$ non-significant and significant at $10 \%, 5 \%$, and $1 \%$, respectively.

De maneira geral, entre os substratos utilizados para a produção de mudas de catingueira, S e SA proporcionaram às mudas maiores valores de MMST, MMSF e AF. As mudas apresentaram crescimento significativo em resposta ao sombreamento apenas na primeira avaliação, aos 30 DAEE, e os maiores níveis de sombreamento induziram maior MMST1, MMSF1 e AF1 (Tabelas 3, 4 e 5).

A taxa de crescimento absoluto (TCA) pode ser usada para se ter ideia da velocidade média de crescimento ao longo do período de observação (BENINCASA, 1988). Para as mudas de catingueira, a TCA calculada para o período entre 30 e 60 DAEE (TCA1) foi semelhante em todos os sombreamentos testados. No entanto, as mudas que se desenvolveram no substrato SA apresentaram maior TCA1, diferindo estatisticamente daquelas que se desenvolveram em A e SC. No período entre 60 e 100 DAEE, houve maior variação da TCA2 das mudas nos diferentes sombreamentos e substratos, e as mudas que se desenvolveram em substrato SAE e em ambiente sem sombreamento apresentaram maior TCA2 que as demais (Tabela 6).

A taxa de crescimento relativo (TCR) é a medida da rapidez com que uma planta cresce quando comparada com o seu tamanho inicial (BENINCASA, 1988). Apenas para TCR entre 30 e 60 DAEE (TCR 1 ) as mudas apresentaram resposta aos tratamentos impostos, e aquelas submetidas a $30 \%$ de sombreamento apresentaram maior TCR1, diferindo significativamente das mudas submetidas ao maior sombreamento (75\%). O substrato que permitiu maior TCR 1 foi SA, em que a TCR1 das mudas de catingueira diferiram significativamente das que se desenvolveram em SC (Tabela 7).

A taxa assimilatória líquida (TAL) expressa a taxa de fotossíntese líquida, em termos de matéria seca produzida por unidade de área foliar. Dessa forma, a TAL indica a eficiência das folhas para produção de biomassa (BENINCASA, 1988). A TAL das mudas de catingueira apresentou diferença estatística entre os substratos apenas no período entre 60 e 100 DAEE (TAL2), com a ressalva de que o substrato SAE possibilitou às mudas TAL2 muito maior que as demais, diferindo estatisticamente daquelas desenvolvidas nos substratos S e AS (Tabela 7).

Segundo Benincasa (1988), a razão de área foliar (RAF) expressa a área foliar útil para a fotossíntese, sendo relação entre a área foliar responsável pela interceptação da energia luminosa e $\mathrm{CO}_{2}$ e a massa seca total, resultado da fotossíntese, sendo esse um componente morfofisiológico. Desse modo, com o crescimento da planta aumenta a interferência das folhas

R. Árvore, Viçosa-MG, v.33, n.3, p.413-423, 2009 
superiores sobre as inferiores, diminuindo a área foliar útil. Os menores valores de RAF, portanto, indicam maior eficiência das folhas em produção de biomassa. As mudas de catingueira apresentaram resposta significativa para as duas primeiras datas avaliadas (30 DAEE, RAF1 e 60 DAEE, RAF2). ARAF1 das mudas desenvolvidas em $\mathrm{S}$ foi significativamente mais baixa que daquelas desenvolvidas em SA e SAE. No entanto, as mudas apresentaram RAF2 mais baixa em SA. À medida que foi maior o nível de sombreamento a que as mudas foram submetidas, A RAF2 delas diminuiu (Tabela 8).

A área foliar específica (AFE) é expressa pela razão entre a área foliar e a massa seca das folhas. A área foliar é um componente morfofisiológico e a massa, componente anatômico de uma espécie vegetal, pois está relacionado à composição interna (número e tamanho) das células do mesófilo. Infere-se daí que o inverso da AFE reflete a espessura das folhas (BENINCASA, 1988). Ferreira (1996) relatou que decréscimos na AFE indicam aumento na espessura da folha resultante do aumento do tamanho e do número de células nas plantas. A AFE aos 60 DAEE (AFE2) das mudas de catingueira foi mais baixa em mudas que cresceram em SC e SA; portanto, pode-se inferir que essas apresentaram maior espessura de folhas. $\mathrm{O}$ sombreamento das mudas não interferiu na sua AFE (Tabela 9).

A razão de peso foliar aos 100 DAEE (RPF3) foi mais alta em mudas que se desenvolveram em SAE (Tabela 9). Considerando que as folhas são a fonte (centro de produção de matéria seca) e que os drenos dependem da exportação dos açúcares produzidos nas folhas (BENINCASA, 1988), a RPF das mudas de catingueira expressa a fração de matéria seca retida nas folhas e não exportada para os demais órgãos. Pode-se inferir, portanto, que as mudas que se desenvolveram em SAE exportaram menos fotoassimilados que as demais, e as desenvolvidas em S e SA apresentaram maior exportação das folhas para caule e raízes.

Scalon et al. (2003), trabalhando com mudas de Bombacopsis glabra (Pasq.) A. Robyns (castanhado-maranhão) sob diferentes intensidades luminosas, não verificaram diferença significativa entre os tratamentos luminosos, no entanto encontrou valores das taxas de crescimento superiores aos das mudas cultivadas sob $50 \%$ de sombreamento.

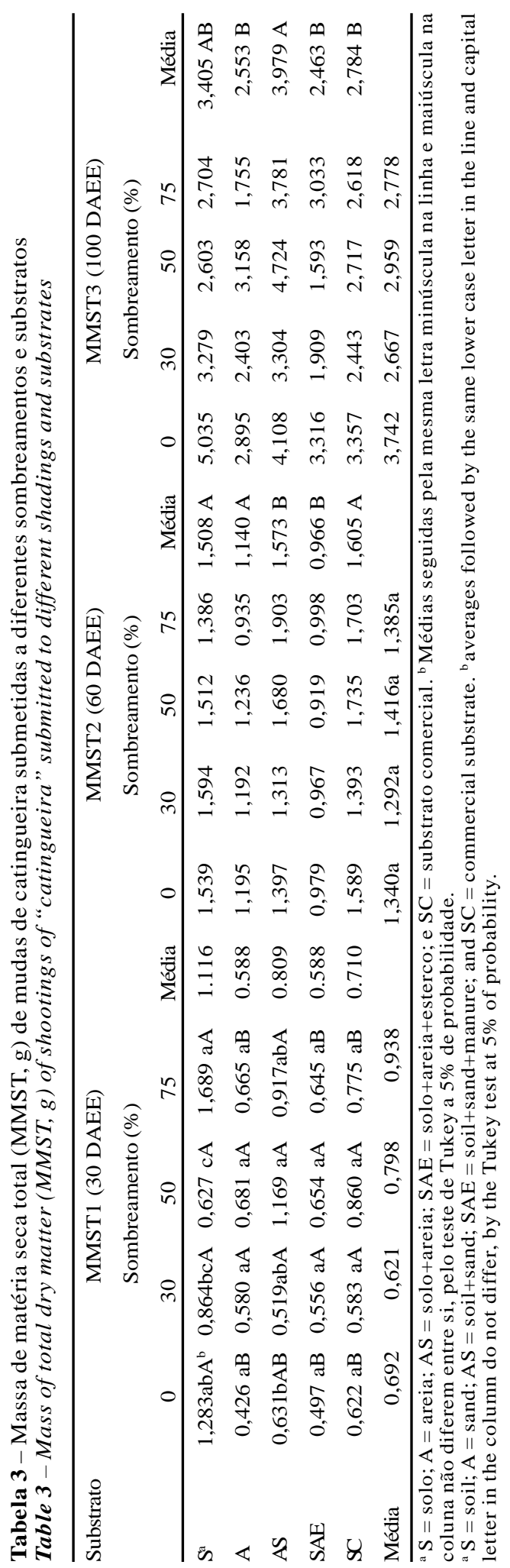


Tabela 4 - Massa de matéria seca de folhas ( MMSF, g) de mudas de catingueira submetidas a diferentes sombreamentos e substratos Table 4-Mass of leaf dry matter ( MMSF, $g$ ) of seedlings of "catingueira" submitted to different shadings and substrates

\begin{tabular}{|c|c|c|c|c|c|c|c|c|c|c|c|c|c|c|c|}
\hline \multirow[t]{3}{*}{ Substrato } & \multicolumn{5}{|c|}{ MMSF1 (30 DAEE) } & \multicolumn{5}{|c|}{ MMSF2 (60 DAEE) } & \multirow{2}{*}{\multicolumn{4}{|c|}{ MMSF3 (100 DAEE) }} & \multirow[b]{3}{*}{ Média } \\
\hline & \multicolumn{5}{|c|}{ Sombreamento $(\%)$} & \multicolumn{5}{|c|}{ Sombreamento (\%) } & Sombreamento (\%) & & & & \\
\hline & 0 & 30 & 50 & 75 & Média & 0 & 30 & 50 & 75 & Média & 0 & 30 & 50 & 75 & \\
\hline A & 0,104 & 0,112 & 0,146 & 0,176 & $0,237 \mathrm{AB}$ & 4,440 & 0,458 & 0,411 & 0,524 & $0,571 \mathrm{BC}$ & 1,058 & 0,853 & 1,193 & 0,652 & 0,939B \\
\hline AS & 0,212 & 0,191 & 0,289 & 0,240 & $0,361 \mathrm{AB}$ & 0,806 & 0,642 & 0,730 & 0,890 & $0,846 \mathrm{~A}$ & 1,879 & 1,594 & 2,655 & 1,485 & $1,903 \mathrm{~A}$ \\
\hline SAE & 0,249 & 0,169 & 0,317 & 0,442 & $0,226 \mathrm{~B}$ & 0,728 & 0,575 & 1,007 & 1,073 & $0,458 \mathrm{C}$ & 1,392 & 0,892 & 0,672 & 1,499 & $1,114 \mathrm{~B}$ \\
\hline Média & $0,227 \mathrm{~b}$ & $0,253 \mathrm{ab}$ & $0,350 \mathrm{ab}$ & $0,377 \mathrm{a}$ & & $0,682 \mathrm{a}$ & $0,630 \mathrm{a}$ & $0,709 \mathrm{a}$ & $0,734 \mathrm{a}$ & & $1,541 \mathrm{a}$ & $1,138 \mathrm{a}$ & $1,353 \mathrm{a}$ & $1,147 \mathrm{a}$ & \\
\hline
\end{tabular}

${ }^{a} \mathrm{~S}=$ solo; $\mathrm{A}=$ areia; $\mathrm{AS}=$ solo+areia; $\mathrm{SAE}=$ solo+areia+esterco; e $\mathrm{SC}=$ substrato comercial. ${ }^{\mathrm{b}}$ Médias seguidas pela mesma letra minúscula na linha e maiúscula na coluna não diferem entre si, pelo teste de Tukey a $5 \%$ de probabilidade.

$\mathrm{S}=$ soil; $\mathrm{A}=$ sand; $\mathrm{AS}=$ soil+sand; $\mathrm{SAE}=$ soil+sand+manure; and $\mathrm{SC}=$ commercial substrate. ${ }^{\mathrm{b}}$ averages followed by the same lower case letter in the line and capital letter in the column do not differ, by the Tukey test at $5 \%$ of probability.

Tabela 5 - Área foliar $\left(\mathrm{AF}, \mathrm{dm}^{2}\right)$ de mudas de catingueira submetidas a diferentes sombreamentos e substratos Table 5 - Leaf area $\left(A F, d m^{2}\right)$ of seedlings of "catingueira" submitted to differents shadings and substrates

\begin{tabular}{|c|c|c|c|c|c|c|c|c|c|c|c|c|c|c|c|}
\hline \multirow[t]{3}{*}{ Substrato } & \multirow{2}{*}{\multicolumn{6}{|c|}{$\begin{array}{c}\text { AF1 (30 DAEE) } \\
\text { Sombreamento (\%) }\end{array}$}} & \multirow{2}{*}{\multicolumn{4}{|c|}{$\begin{array}{c}\text { AF2 (60 DAEE) } \\
\text { Sombreamento (\%) }\end{array}$}} & \multirow{2}{*}{\multicolumn{5}{|c|}{$\begin{array}{l}\text { AF3 (100 DAEE) } \\
\text { Sombreamento (\%) }\end{array}$}} \\
\hline & & & & & & & & & & & & & & & \\
\hline & 0 & 30 & 50 & 75 & Média & 0 & 30 & 50 & 75 & Média & 0 & 30 & 50 & 75 & Média \\
\hline$S^{a}$ & 31,536 & 30,284 & 39,770 & 45,202 & $66,90 \mathrm{~A}^{\mathrm{b}}$ & 85,862 & 107,968 & 114,362 & 97,340 & $131,57 \mathrm{AB}$ & 217,828 & 194,146 & 161,326 & 162,883 & $185,16 \mathrm{~B}$ \\
\hline A & 49,862 & 43,384 & 43,640 & 54,178 & $36,70 \mathrm{C}$ & 70,540 & 79,878 & 78,534 & 78,534 & $106,05 \mathrm{BC}$ & 134,260 & 136,934 & 179,960 & 134,128 & $146,32 B$ \\
\hline AS & 51,128 & 61,720 & 72,640 & 65,770 & $74,30 \mathrm{~A}$ & 110,402 & 114,302 & 133,376 & 118,014 & $149,58 \mathrm{~A}$ & 232,104 & 260,622 & 427,752 & 267,412 & $296,97 \mathrm{~A}$ \\
\hline SAE & 59,068 & 55,582 & 87,302 & 95,238 & $47,77 \mathrm{BC}$ & 108,628 & 100,958 & 194,284 & 194,464 & $83,70 \mathrm{C}$ & 204,618 & 147,748 & 110,994 & 260,806 & $181,04 \mathrm{~B}$ \\
\hline $\mathrm{SC}$ & 44,664 & 70,224 & 64,480 & 88,184 & $62,81 \mathrm{AB}$ & 118,720 & 141,662 & 132,682 & 133,218 & $119,02 \mathrm{ABC}$ & 122,412 & 168,450 & 199,186 & 193,020 & $170,77 \mathrm{~B}$ \\
\hline Média & $47,25 b$ & $52,24 \mathrm{~b}$ & $61,57 \mathrm{ab}$ & $69,71 \mathrm{a}$ & & $102,56 a$ & $108,95 \mathrm{a}$ & $130,65 a$ & $129,78 \mathrm{a}$ & & $182,24 \mathrm{a}$ & $181,58 \mathrm{a}$ & $215,84 \mathrm{a}$ & $205,35 \mathrm{a}$ & \\
\hline
\end{tabular}

${ }^{a} \mathrm{~S}=$ solo; $\mathrm{A}=$ areia; $\mathrm{AS}=$ solo+areia; $\mathrm{SAE}=$ solo+areia+esterco; e $\mathrm{SC}$ = substrato comercial. ${ }^{\mathrm{b}}$ Médias seguidas pela mesma letra minúscula na linha e maiúscula na coluna não diferem entre si, pelo teste de Tukey a $5 \%$ de probabilidade.

${ }^{a} \mathrm{~S}=$ soil; $\mathrm{A}=$ sand; $\mathrm{AS}=$ soil+sand; $\mathrm{SAE}=$ soil+sand+manure; and $\mathrm{SC}=$ commercial substrate. ${ }^{\mathrm{b}}$ averages followed by the same lower case letter in the line and capital letter in the column do not differ, by the Tukey test at $5 \%$ of probability. 
Tabela 6 - Taxa de crescimento absoluto (TCA, mg.dia ${ }^{-1}$ ) de mudas de catingueira submetidas a diferentes sombreamentos e substratos

Table 6-Absolute growth rate (TCA, $m$ g.day ${ }^{-1}$ ) of seedlings of "catingueira" submitted to different shadings and substrates

\begin{tabular}{lcccccccccc}
\hline Substrato & \multicolumn{4}{c}{ TCA 1 (30-60 DAEE) } & \multicolumn{5}{c}{ TCA2 (60-100 DAEE) } \\
& \multicolumn{4}{c}{ Sombreamento (\%) } & \multicolumn{4}{c}{ Sombreamento (\%) } \\
& 0 & 30 & 50 & 75 & Média & 0 & 30 & 50 & 75 & Média \\
\hline $\mathrm{S}^{\mathrm{a}}$ & 23,323 & 18,543 & 16,832 & 8,161 & $16,715 \mathrm{AB}^{\mathrm{b}}$ & 42,490 & 30,275 & 48,035 & 20,505 & $35,326 \mathrm{AB}$ \\
$\mathrm{A}$ & 14,601 & 12,456 & 8,027 & 10,700 & $11,446 \mathrm{~B}$ & 58,420 & 23,550 & 16,860 & 50,880 & $37,428 \mathrm{AB}$ \\
$\mathrm{AS}$ & 29,311 & 24,553 & 26,507 & 28,123 & $27,123 \mathrm{~A}$ & 44,205 & 26,235 & 24,555 & 22,870 & $29,466 \mathrm{~B}$ \\
$\mathrm{SAE}$ & 23,231 & 24,057 & 15,479 & 29,893 & $23,165 \mathrm{AB}$ & 67,765 & 49,770 & 76,100 & 46,950 & $60,146 \mathrm{~A}$ \\
$\mathrm{SC}$ & 7,761 & 22,112 & 26,794 & $-9,177$ & $11,872 \mathrm{~B}$ & 87,395 & 42,120 & 32,935 & 27,290 & $47,435 \mathrm{AB}$ \\
Média & $19,645 \mathrm{a}$ & $20,344 \mathrm{a}$ & $18,728 \mathrm{a}$ & $13,540 \mathrm{a}$ & & $60,055 \mathrm{a}$ & $34,390 \mathrm{~b}$ & $38,568 \mathrm{ab}$ & $34,828 \mathrm{~b}$ & \\
\hline
\end{tabular}

${ }^{\text {a }} \mathrm{S}=$ solo; $\mathrm{A}=$ areia $; \mathrm{AS}=$ solo+areia $\mathrm{SAE}=$ solo+areia+esterco; e $\mathrm{SC}=$ substrato comercial. ${ }^{\mathrm{b}}$ Médias seguidas pela mesma letra minúscula na linha e maiúscula na coluna não diferem entre si, pelo teste de Tukey a $5 \%$ de probabilidade.

${ }^{\mathrm{a}} \mathrm{S}=$ soil $; \mathrm{A}=$ sand; $\mathrm{AS}=$ soil+sand; $\mathrm{SAE}=$ soil+sand+manure; and $\mathrm{SC}=$ commercial substrate. ${ }^{\mathrm{b}}$ averages followed by the same lower case letter in the line and capital letter in the column do not differ, by the Tukey test at $5 \%$ of probability.

Tabela 7 - Taxa de crescimento relativo (TCR, mg. $\mathrm{mg}^{-1} \cdot \mathrm{dia}^{-1}$ ) e taxa assimilatória líquida (TAL, mg. $\left.\mathrm{dm}^{-2} \cdot \mathrm{dia}^{-1}\right)$ de mudas de catingueira submetidas a diferentes sombreamentos e substratos

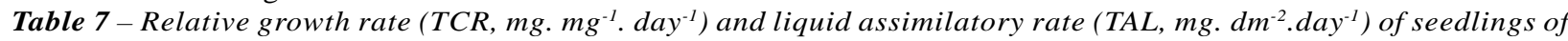
"catingueira" submitted to different shadings and substrates

\begin{tabular}{lcccccccccc}
\hline Substrato & \multicolumn{4}{c}{ TCR1 (30-60 DAEE) } & \multicolumn{5}{c}{ TAL2 (60-100 DAEE) } \\
& \multicolumn{4}{c}{ Sombreamento (\%) } & \multicolumn{4}{c}{ Sombreamento (\%) } \\
& 0 & 30 & 50 & 75 & Média & 0 & 30 & 50 & 75 & Média \\
\hline$S^{\mathrm{a}}$ & 0,0288 & 0,0250 & 0,0172 & 0,0078 & $0,0197 \mathrm{AB}^{\mathrm{b}}$ & 0,0657 & 0,1315 & 0,2748 & 0,4397 & $0,2279 \mathrm{~B}$ \\
$\mathrm{~A}$ & 0,0218 & 0,0176 & 0,0088 & 0,0132 & $0,0154 \mathrm{AB}$ & 1,36226 & 0,18178 & 0,25428 & 0,82246 & $0,6552 \mathrm{AB}$ \\
$\mathrm{AS}$ & 0,0278 & 0,0266 & 0,0224 & 0,0256 & $0,0256 \mathrm{~A}$ & $-0,03816$ & 0,3682 & 0,2315 & 0,2315 & $0,1586 \mathrm{~B}$ \\
$\mathrm{SAE}$ & 0,0238 & 0,0292 & 0,0136 & 0,0226 & $0,0223 \mathrm{AB}$ & 1,73916 & 1,2566 & 1,83668 & 0,3671 & $1,300 \mathrm{~A}$ \\
$\mathrm{SC}$ & 0,0080 & 0,0188 & 0,0264 & $-0,0020$ & $0,0128 \mathrm{~B}$ & 1,3944 & 0,5208 & 0,1317 & $-0,06296$ & $0,4960 \mathrm{AB}$ \\
Média & $0,0220 \mathrm{ab}$ & $0,0234 \mathrm{a}$ & $0,0177 \mathrm{ab}$ & $0,0134 \mathrm{~b}$ & & $0,9047 \mathrm{a}$ & $0,4918 \mathrm{a}$ & $0,5458 \mathrm{a}$ & $0,3279 \mathrm{a}$ & \\
\hline
\end{tabular}

a $\mathrm{S}=$ solo; $\mathrm{A}=$ areia $; \mathrm{AS}=$ solo+areia $; \mathrm{SAE}=$ solo+areia+esterco; e $\mathrm{SC}=$ substrato comercial. ${ }^{\mathrm{b}}$ Médias seguidas pela mesma letra minúscula na linha e maiúscula na coluna não diferem entre si, pelo teste de Tukey a $5 \%$ de probabilidade.

${ }^{\text {a }} \mathrm{S}=$ soil $; \mathrm{A}=$ sand; $\mathrm{AS}=$ soil+sand $\mathrm{SAE}=$ soil+sand+manure; and $\mathrm{SC}=$ commercial substrate. ${ }^{\mathrm{b}}$ averages followed by the same lower case letter in the line and capital letter in the column do not differ, by the Tukey test at $5 \%$ of probability.

Tabela 8 - Razão de área foliar (RAF, $\left.\mathrm{dm}^{2} \cdot \mathrm{mg}^{-1}\right)$ de mudas de catingueira submetidas a diferentes sombreamentos e substratos Table 8 - Leaf area ratio $\left(R A F, d m^{2} . m^{-1}\right)$ of seedlings of "catingueira" submitted to different shadings and substrates

\begin{tabular}{|c|c|c|c|c|c|c|c|c|c|c|}
\hline \multirow[t]{2}{*}{ Substrato } & \multicolumn{5}{|c|}{$\begin{array}{l}\text { RAF1 (30 DAEE) } \\
\text { Sombreamento (\%) }\end{array}$} & \multicolumn{5}{|c|}{$\begin{array}{l}\text { RAF2 (60 DAEE) } \\
\text { Sombreamento (\%) }\end{array}$} \\
\hline & 0 & 30 & 50 & 75 & Média & 0 & 30 & 50 & 75 & Média \\
\hline$S^{\mathrm{a}}$ & 0,0697 & 0,0648 & 0,0605 & 0,0679 & $0,0658 \mathrm{C}^{\mathrm{b}}$ & 0,0999 & 0,0915 & 0,0885 & 0,1087 & $0,0971 \mathrm{~A}$ \\
\hline A & 0,1060 & 0,0811 & 0,0673 & 0,0836 & $0,0845 \mathrm{ABC}$ & 0,0692 & 0,0830 & 0,0842 & 0,1033 & $0,0849 \mathrm{AB}$ \\
\hline AS & 0,0807 & 0,1157 & 0,0862 & 0,0819 & $0,0911 \mathrm{AB}$ & 0,0677 & 0,0841 & 0,0763 & 0,0731 & 0,0753 В \\
\hline SAE & 0,0953 & 0,1128 & 0,0968 & 0,1040 & $0,1022 \mathrm{~A}$ & 0,0753 & 0,0765 & 0,1154 & 0,1011 & $0,0921 \mathrm{AB}$ \\
\hline $\mathrm{SC}$ & 0,0424 & 0,0837 & 0,1008 & 0,0664 & $0,0733 \mathrm{BC}$ & 0,0713 & 0,0885 & 0,0903 & 0,0962 & $0,0866 \mathrm{AB}$ \\
\hline Média & $0,0788 \mathrm{a}$ & $0,0916 \mathrm{a}$ & $0,0823 \mathrm{a}$ & $0,0808 \mathrm{a}$ & & $0,0767 b$ & $0,0847 \mathrm{ab}$ & $0,0911 \mathrm{ab}$ & $0,0965 \mathrm{a}$ & \\
\hline
\end{tabular}

${ }^{a} \mathrm{~S}=$ solo; $\mathrm{A}=$ areia $; \mathrm{AS}=$ solo+areia $; \mathrm{SAE}=$ solo+areia+esterco; e $\mathrm{SC}=$ substrato comercial. ${ }^{\mathrm{b}}$ Médias seguidas pela mesma letra minúscula na linha e maiúscula na coluna não diferem entre si, pelo teste de Tukey a $5 \%$ de probabilidade.

${ }^{a} \mathrm{~S}=$ soil $; \mathrm{A}=$ sand; $\mathrm{AS}=$ soil+sand; $\mathrm{SAE}=$ soil+sand+manure; and $\mathrm{SC}=$ commercial substrate. ${ }^{\mathrm{b}}$ averages followed by the same lower case letter in the line and capital letter in the column do not differ, by the Tukey test at $5 \%$ of probability. 
Tabela 9 - Área foliar específica (AFE, $\mathrm{dm}^{2} \cdot \mathrm{mg}^{-1}$ ) e razão de peso foliar (RPF, $\mathrm{mg} \cdot \mathrm{mg}^{-1}$ ) de mudas de catingueira submetidas a diferentes sombreamentos e substratos

Table 9 - Specific leaf area $\left(A F E, d^{2} . m^{-1}\right)$ and foliar weight ratio $\left(R P F, m g . m g^{-1}\right)$ of seedlings of “catingueira” submitted to different shadingse substrates

\begin{tabular}{lcccccccccc}
\hline Substrato & \multicolumn{4}{c}{ AFE2 (30 DAEE) } & \multicolumn{4}{c}{ RPF3 (100 DAEE) } \\
& 0 & 30 & 50 & 75 & Média & 0 & 30 & 50 & 75 & Média \\
\hline$S^{\mathrm{a}}$ & 0,2731 & 0,1813 & 0,1994 & 0,2147 & $0,2171 \mathrm{~A}^{\mathrm{b}}$ & 0,3589 & 0,3367 & 0,3900 & 0,3402 & $0,3564 \mathrm{~B}$ \\
$\mathrm{~A}$ & 0,1605 & 0,1771 & 0,1924 & 0,2037 & $0,1834 \mathrm{AB}$ & 0,3765 & 0,4560 & 0,4261 & 0,4501 & $0,4272 \mathrm{AB}$ \\
$\mathrm{AS}$ & 0,1361 & 0,1469 & 0,1796 & 0,1858 & $0,1621 \mathrm{~B}$ & 0,3366 & 0,3920 & 0,4004 & 0,3664 & $0,3738 \mathrm{~B}$ \\
$\mathrm{SAE}$ & 0,1489 & 0,1761 & 0,1946 & 0,1805 & $0,1750 \mathrm{AB}$ & 0,4264 & 0,4729 & 0,5570 & 0,3898 & $0,4615 \mathrm{~A}$ \\
$\mathrm{SC}$ & 0,1326 & 0,1618 & 0,1684 & 0,1926 & $0,1638 \mathrm{~B}$ & 0,41124 & 0,4040 & 0,41248 & 0,41308 & $0,4102 \mathrm{AB}$ \\
Média & $0,1702 \mathrm{a}$ & $0,1752 \mathrm{a}$ & $0,1881 \mathrm{a}$ & $0,1877 \mathrm{a}$ & & $0,3819 \mathrm{a}$ & $0,4123 \mathrm{a}$ & $0,4372 \mathrm{a}$ & $0,39191 \mathrm{a}$ & \\
\hline
\end{tabular}

${ }^{a} \mathrm{~S}=$ solo; $\mathrm{A}=$ areia; $\mathrm{AS}=$ solo+areia; $\mathrm{SAE}=$ solo+areia+esterco; e $\mathrm{SC}=$ substrato comercial. ${ }^{\mathrm{b}}$ Médias seguidas pela mesma letra minúscula na linha e maiúscula na coluna não diferem entre si, pelo teste de Tukey a $5 \%$ de probabilidade.

${ }^{a} \mathrm{~S}=$ soil $; \mathrm{A}=$ sand; $\mathrm{AS}=$ soil+sand; $\mathrm{SAE}=$ soil+sand+manure; and $\mathrm{SC}=$ commercial substrate. ${ }^{\mathrm{b}}$ averages followed by the same lower case letter in the line and capital letter in the column do not differ, by the Tukey test at $5 \%$ of probability.

Frequentemente, as análises do crescimento de mudas são utilizadas para predizer o grau de tolerância das diferentes espécies ao sombreamento. Acreditase que as espécies tolerantes apresentam crescimento mais lento em relação às não tolerantes, devido às suas taxas metabólicas mais baixas. O rápido crescimento em altura quando sombreada é um mecanismo de adaptação das plantas competitivas (GRIME, 1965, 1977) ou nômades (TINOCO e VASQUES-YANES, 1985), como forma de escape ao déficit de luz, já que estas não são capazes de tolerar baixas intensidades luminosas através do reajuste de suas taxas metabólicas. Geralmente há grande diversidade de respostas das árvores nativas à luminosidade, principalmente quanto ao desenvolvimento vegetativo da parte aérea e à sobrevivência das mudas (SCALON e ALVARENGA, 1993). Dessa forma, a eficiência do crescimento da planta pode ser relacionada à habilidade de adaptação das plântulas às condições luminosas do ambiente. Cada espécie florestal apresenta exigência luminosa própria para seu desenvolvimento, e algumas plântulas podem aproveitar e se desenvolver melhor em locais com alta intensidade luminosa e outras em sombreamento, existindo ainda aquelas espécies que são intermediárias e as de ampla dispersão (PORTELA et al., 2001). O crescimento satisfatório de algumas espécies em ambientes com diferentes disponibilidades luminosas pode ser atribuído à capacidade de ajustar, eficaz e rapidamente, seu comportamento fisiológico para maximizar a aquisição de recursos nesse ambiente (DIASFILHO, 1997). Pode-se verificar, pelos resultados obtidos neste trabalho, que a catingueira apresenta alguma dependência do sombreamento, apenas nos primeiros
30 dias de desenvolvimento, apresentando maior MMST, MMSF e AF em condições mais sombreadas (Tabelas 2 a 5). A partir disso, o crescimento da catingueira independe do sombreamento a que as mudas estão expostas (Tabelas 2 a 9).

Cunha et al. (2006) verificaram, em mudas de Acacia mangium e Acacia auriculiformis, que o maior crescimento ocorreu em substratos com a presença de esterco bovino nas mudas. Na composição do substrato para o crescimento de plântulas, a fonte orgânica é responsável pela retenção de umidade e fornecimento de parte dos nutrientes. Tradicionalmente, o esterco bovino é utilizado como fonte orgânica na composição de substratos para viveiros de mudas de café, de plantas hortícolas e de plantas arbóreas (FONSECA, 1988; SANTOS et al., 1994; ANDRADE NETO et al., 1999). No entanto, a disponibilidade do esterco bovino de qualidade depende da região e também do manejo das pastagens. Na região do semiárido nordestino, a criação de caprinos e ovinos possibilita que o esterco caprino ou ovino seja abundante para utilização em substratos.

Neste trabalho, as mudas de catingueira apresentaram altas taxas de crescimento em níveis até $50 \%$ de sombreamento, com pouca ou nenhuma diferença entre os diferentes sombreamentos a que foram submetidas. No entanto, os substratos que mais propiciaram o crescimento da catingueira foram os que apresentavam solo (S, AS e SAE), característico das regiões de desenvolvimento da espécie, com destaque para o solo acrescido de esterco caprino.

R. Árvore, Viçosa-MG, v.33, n.3, p.413-423, 2009 
De acordo com Maia (2004), a catingueira é caracterizada como uma das espécies de mais ampla dispersão no semiárido nordestino, tanto na luminosidade quanto no substrato, de grande adaptação às diferentes situações as quais é submetida.

\section{CONCLUSÕES}

Nas condições deste trabalho, foi possível concluir que as mudas de catingueira (Caesalpinia pyramidalis Tul.), em geral, não foram influenciadas pelo sombreamento ao qual elas foram submetidas e que solo coletado em região de caatinga, combinado ou não, com areia e esterco, possibilitou maior crescimento das mudas.

A produção de mudas de catingueira (Caesalpinia pyramidalis Tul.) pode ser realizada em viveiros sem cobertura de telas tipo sombrite, e o substrato utilizado deve conter material de solo coletado da caatinga.

\section{REFERÊNCIAS}

AMO, S. R. Alguns aspectos de la influencia de la luz sobre el crescimento de estados juveniles de especies primarias. In: GOMEZ-POMPA, A.; AMO, S. R. Investigaciones sobre la regeneración de selvas altas en Veracruz, México. México: Alhambra Mexicana, 1985. v.2. p.79-92.

ANDRADE NETO, A.; MENDES, A. N. G.; GUIMARÃES, P. T. G. Avaliação de substratos alternativos e tipo de adubação para a produção de mudas de cafeeiro em tubetes. Ciência e Agrotecnologia, v.23, n.2, p.270-280, 1999.

BAHIA. Inventário de Plantas Medicinais do Estado da Bahia. LOCAL: SEPLANTEC/CADCT, 1979. 25p.

BEnINCASA, M. M. P. Análise de crescimento de plantas: noções básicas. Jaboticabal: FUNEP, 1988. 41p.

CARneiro, J. G. A. Produção e controle de qualidade de mudas florestais. Curitiba: UFPR:FUPEF; Campos: UENF, 1995. 451p.

CUNHA, A. M. et al. Efeito de diferentes substratos sobre o desenvolvimento de mudas de Acacia sp. Revista Árvore, v.30, n.2, p.207-214, 2006.

R. Árvore, Viçosa-MG, v.33, n.3, p.413-423, 2009
DIAS FILHO, M. B. Physiological response of Solanum crinitum Lam. to contrasting light environments. Pesquisa Agropecuária Brasileira, v.32, n.6, p.789-796, 1997.

FERREIRA, E. Ajustamento osmótico e análise de crescimento de plantas de milho (Zea mays L.), em função do nível de potássio e estresse hídrico. 1996. FOLHAS. Tese (Doutorado emBotânica) Universidade Estadual Paulista, Botucatu, 1996.

FONSECA, E. P. Efeito de diferentes substratos na produção de mudas de Eucalyptus grandis W. Hill ex Maiden em “Win-strip”. 1988. 81f. Dissertação (Mestrado em Ciência Florestal) - Universidade Federal de Viçosa, Viçosa, MG, 1988.

FONSECA, T. G. Produção de mudas de hortaliças em substratos de diferentes composições com adição de $\mathrm{CO}_{2}$ na água de irrigação. 2001. 72f. Dissertação (Mestrado em Agronomia) - Escola Superior de Agricultura Luiz de Queiroz, Piracicaba, 2001.

GAJEGO, E. B. et al. Crescimento de plantas jovens de Maclura tinctoria e Hymenaea courbaril em diferentes condições de sombreamento. In: CONGRESSO NACIONAL DE FISIOLOGIA, 8., 2001, Ilhéus. Anais... Ilhéus: 2001. CD-ROM.

GARCIA, C. H. Tabelas para classificação do coeficiente de variação. Piracicaba: IPEF, 1989. 12p. (Circular Técnica, 171).

GRIME, J. P. Evidence for the existence of three primary srategies in plants and its relevance to ecological and evolutionary theory. The American Naturalist, v.982, n.3, p.1169-1194, 1977.

GRIME, J. P. Shade tolerance in flowering plants. Nature, v.5006, n.208, p.161-163, 1965.

HARDESTY, L. H.; BOX, T. W.; MALECHEK, J. C. Season of cutting affects bio mass production by coppicing browse species of the Brazilian caatinga. Journal of Range Management, v.41, n.6, p.447-80, 1988.

IPEF. Reflorestamento. Disponível em: <http.www.ipef.com.br>, Acesso em: 12 jan. de 2005. 
KAMPF, A. N. Produção comercial de plantas ornamentais. Guaíba:

Agropecuária, 2000. 254p.

LIMA, J. L. S. Plantas forrageiras das caatingas: usos e potencialidades. Petrolina: Embrapa Semi-Arido/ PNE/ RBG-KEW, 1996. 44p.

MAGALHÃES, A. C. N. Análise quantitativa de crescimento. In: FERRI, M. G. Fisiologia vegetal. São Paulo: EDUSP, 1986. p.331-350.

MAIA, G. N. Caatinga: árvores arbustos e suas utilidades. São Paulo: Leitura e Arte, 2004. 413p.

NEGREIROS, J. R. S. Diferentes substratos na formação de mudas de maracujazeiro-amarelo. Revista Ceres, v.51, n.294, p.243-343, 2004.

NEGREIROS, J. R. S. Diferentes substratos na formação de mudas de maracujazeiro-amarelo. Revista Ceres, v.51, n.294, p.243-343, 2004.

NISHIZAWA, T.; TSUCHIYA, A.; PINTO, M. M. $\mathrm{V}$. Characteristics and utilization of tree species in the semi-arid woodland of north-east Brazil In: NISHIZAWA, T.; UITTO, J. I. The fragile tropics of Latin America: sustainable management of changing environments.

Disponível em: <http://www.unu.edu/unupress/ unupbooks/80877e/80877E00.htm\#Contents>, Acesso em: 05 mar. de 2005.

OLIVEIRA, O. F. Caatinga. Mossoró: ESAM, 1976. 86p.

OLIVEIRA, R. P. et al. Mudas de citros. Pelotas: Embrapa Clima Temperado, 2005. (Sistema de Produção, 1) Disponível em: <http:// www.cpact.embrapa.br/sistemas/mudas/index.htm >. Acesso em: 26 set. de 2006.

PFEISTER, J. A.; MALECHEK, J. C. Dietary selection by goats and sheep in a deciduous woodland of Northeastern Brazil. Journal of Range Management, v. 39, n. 1, p. 24-28, 1986.
PORTELA, R. C. Q.; SILVA, I. L.; PINÃRODRIGUES, F. C. M. Crescimento inicial de mudas de Clitória fairchildiana Howard e Peltophorum dubium (Sprenge) Taub em diferentes condições de sombreamento. Ciência Florestal, v.11, n.2, p.163-170, 2001.

SAMPAIO, E.; RODAL, M. J. Biodiversidade da caatinga. Disponível em: $<$ http// www.biodiversitas.org/caatinga>, Acesso em: 12 jan. de 2000 .

SANTOS, L. P.; CARVALHO, M. M.; CARVALHO, J. G. Efeitos de doses de nitrato de potássio e esterco de curral na composição do substrato para a formação de cafeeiro. Ciência e Prática, v.18, n.1, p.42-48, 1994.

SCALON, S. P. Q.; ALVARENGA, A. A. Efeito do sombreamento sobre a formação de mudas de pau-pereira (Platycyamus regnelli Benth.).

Revista Árvore, v. 17, n.3, p.265-270, 1993.

SCALON, S. P. Q. et al. Crescimento inicial de mudas de Bombacopsis glabra (Pasq.) A. Robyns sob condição de sombreamento. Revista Árvore, v.27, n.6, p.753-758, 2003.

SILVEIRA, R. L. V. et al. Adubação e nutrição de espécies nativas: viveiro e campo. São Paulo: Universidade São Paulo, 2002. 22p.

TINOCO, C.; VASQUEZ-YANES, C. Diferencias en poblaciones de Piper hispidus hajo condiciones de luz contratante en uma selva alta perenifolia. In: GOMEZ-POMPA, A.; AMO, R. S. (Eds.) Investigaciones sobre la regeneration de selvas altas em Vera Cruz. México: Alhambra Mexicana, 1985. Tomo 2. p.267-281.

WILSON, C. G. S. Tomato production in bark substrates. Acta Horticulturae, v. 150 , p.271-276, 1983. 
